\title{
marges Marges
}

revue d'art contemporain Revue d'art contemporain

16 | 2013

\section{Art contemporain et Cultural studies}

\section{dOCUMENTA (13)}

Kassel, 9 juin - 16 septembre 2012

\section{Jérôme Glicenstein}

\section{(2) OpenEdition}

\section{Journals}

Édition électronique

URL : http://journals.openedition.org/marges/277

DOI : $10.4000 /$ marges. 277

ISSN : 2416-8742

Éditeur

Presses universitaires de Vincennes

\section{Édition imprimée}

Date de publication : 15 mars 2013

Pagination : 132-133

ISBN : 978-2-84292-366-2

ISSN : 1767-7114

Référence électronique

Jérôme Glicenstein, « dOCUMENTA (13) », Marges [En ligne], 16 | 2013, mis en ligne le 15 mars 2014, consulté le 22 septembre 2020. URL : http://journals.openedition.org/marges/277 ; DOI : https:// doi.org/10.4000/marges. 277 


\section{dOCUMENTA (13)}

Kassel, 9 juin - 16 septembre 2012

La $13^{\mathrm{e}}$ édition de la Documenta pourrait faire figure d'événement polymorphe et foisonnant, défiant toute description synthétique. Elle était éclatée en une infinité de lieux, artistiques ou non - parcs, tunnels, banque, hôtel, ex-future mosquée, vigne, gare, magasins, bibliothèques, musées en tout genre -, sans même mentionner les lieux habituels tels que le Fridericianum ou la DocumentaHalle. En outre, à l'instar de l'avant-dernière édition (2002), elle ne se limitait pas à la ville de Kassel puisque d'autres événements étaient signalés à Kaboul, Alexandrie et Banff. Les deux dernières villes citées étaient sans doute assez anecdotiques (il s'agissait principalement d'organiser des workshops en compagnie d'une partie des artistes); pour ce qui est de Kaboul, les choses étaient différentes puisqu'une version réduite de l'exposition de Kassel y avait été présentée. Cette insistance sur le lien entre Kassel et Kaboul était sans doute l'élément le plus identifiable de la Documenta. Il avait d'ailleurs été mis en avant par la commissaire, Carolyn Christov-
Bakargiev, dans les différents textes de présentation. Quelques artistes afghans avaient ainsi été invités à Kassel et certains artistes occidentaux avaient orienté leur production autour de thématiques « afghanes».

En dépit de la diversité apparente des pratiques, d'autres éléments récurrents apparaissaient ici ou là. Et en premier lieu, une forme de retour aux sources de la Documenta, avec l'idée selon laquelle l'une de ses missions serait de revisiter l'histoire de l'art en donnant à voir des œuvres méconnues ou oubliées. Une quinzaine d'artistes du siècle passé, parfois disparus depuis de nombreuses années, avaient ainsi fait l'objet de présentations spécifiques. C'était le cas de Charlotte Salomon, Hannah Ryggen, Emily Carr, Margaret Preston, Maria Martins, Konrad Zuse, Korbinian Aigner, Antoni Cumella, etc. D'autres, plus contemporains quoique déjà âgés ou décédés, avaient également fait l'objet de présentations spécifiques, sans doute avec la volonté de permettre une réévaluation de leurs œuvres. C'était le cas en 
particulier de Gustav Metzger, dont le travail des années 1950 faisait l'objet d'une présentation extensive à la Documenta-Halle, mais aussi d'artistes marginaux des années 1960 et 1970: Thomas Bayrle, Llyn Foulkes, Mark Lombardi, Etel Adnan, Fabio Mauri, Aase Texmon Rygh, Tamas St. Turba ou Anna Boghiguian. La même volonté de revalorisation de pratiques oubliées ou marginalisées s'étendait à quelques artistes non-occidentaux, notamment des Aborigènes d'Australie, mais aussi dans un registre complètement opposé, à des peintres de guerre de l'armée ViêtCong. Il y avait un cas à part : celui d'Alighiero Boetti. Ce dernier, qui était loin d'être un artiste marginal, avait séjourné en Afghanistan au début des années 1970. La présentation de son travail permettait ainsi de proposer un élément de structuration à l'ensemble: entre Orient et Occident, art moderne et art contemporain, œuvres et documents.

Si l'on met de côté l'idée d'un axe Kassel/Kaboul, la manifestation était pourtant difficile à circonscrire. La Documenta 13 semblait surtout opérer une synthèse entre les différents partis pris des manifestations précédentes. Certes, il n'y avait pas d'orientation politique aussi lisible qu'au cours des quinze dernières années, ni d'allégeance au marché de l'art comme cela avait pu être le cas au cours des années 1980. Il n'y avait pas non plus de parti pris curatorial fort, à la différence des éditions dirigées par Harald Szeemann, Jan Hoet, Catherine David ou Okwui Enwezor. Il y avait néanmoins un peu de tout cela: quelques œuvres à contenu politique (Critical Art Ensemble, CAMP, AND, AND, AND), quelques œuvres d'artistes historiques (Salvador Dalí, Giorgio Morandi, Julio Gonzalez) ou contemporains déjà bien établis: Giuseppe Penone, Lawrence Weiner, Joan Jonas, Jimmy Durham, Rosemarie Trockel, William Kentridge), quelques œuvres des vedettes du moment (Walid Raad, Tino Sehgal, Ryan Gander, Roman Ondak, Julie Mehretu, Anri Sala, Jennifer Allora et Guillermo Calzadilla...). Le tout accompagné d'un tout petit nombre d'artistes émergents.

Cet esprit de synthèse, qui définit sans doute le mieux l'ensemble de l'exposition, se retrouvait emblématiquement dans l'installation The Brain, la pièce maîtresse de la Documenta. Ce « cerveau » rassemblait au centre du Fridericianum un ensemble d'éléments sans lien apparent entre eux, si ce n'est l'idée qu'ils renverraient tous aux choix curatoriaux. Il y avait là une cinquantaine d'objets: des photographies de Lee Miller se baignant dans la salle de bains d'Hitler, des bouteilles utilisées comme modèles par Giorgio Morandi, la maquette d'un des premiers ordinateurs, des poteries paraguayennes, des sculptures fondues lors d'un bombardement à Beyrouth, différentes versions de l'Objet à détruire de Man Ray, une peinture de Gustav Metzger accidentellement détruite... et des " princesses bactriennes », sculptures de 4000 ans en provenance d'Afghanistan. Ce bric-à-brac censé refléter l'esprit de la Documenta 13 permettait d'envisager différemment les remarques un peu cryptiques formulées par la commissaire dans le catalogue: "dOCUMENTA (13) est dédié à la recherche artistique et aux formes d'imagination qui explorent l'engagement, la matière, les choses, l'incarnation et la vie elle-même en relation (sans subordination) avec la théorie [...] dOCUMENTA (13) suit une vision holiste et non-logocentrique qui est sceptique vis-à-vis de la croyance persistante dans la croissance économique. Cette vision est partagée par et reconnaît les formes et pratiques de connaissance de tous les producteurs du monde, animés et inanimés, y compris les hommes. ".

\section{Jérôme Glicenstein}

